\title{
Long-range forces : atmospheric neutrino oscillation at a magnetized detector
}

\author{
Abhijit Samanta* \\ Ramakrishna Mission Vivekananda University, \\ Belur Math, Howrah 711 202, India \\ and \\ ${ }^{\dagger}$ Harish-Chandra Research Institute, \\ Chhatnag Road, Jhusi, Allahabad 211 019, India
}

(Dated: June 18, 2021)

\begin{abstract}
Among the combinations $L_{e}-L_{\mu}, L_{e}-L_{\tau}$ and $L_{\mu}-L_{\tau}$ any one can be gauged in anomaly free way with the standard model gauge group. The masses of these gauge bosons can be so light that it can induce long-range forces on the Earth due to the electrons in the Sun. This type of forces can be constrained significantly from neutrino oscillation. As the sign of the potential is opposite for neutrinos and antineutrinos, a magnetized iron calorimeter detector (ICAL) would be able to produce strong constraint on it. We have made conservative studies of these long-range forces with atmospheric neutrinos at ICAL considering only the muons of charge current interactions. We find stringent bounds on the couplings $\alpha_{e \mu, e \tau} \lesssim 1.65 \times 10^{-53}$ at $3 \sigma$ CL with an exposure of 1 Mton.yr if there is no such force. For nonzero input values of the couplings we find that the potential $V_{e \mu}$ opposes and $V_{e \tau}$ helps to discriminate the mass hierarchy. However, both potentials help significantly to discriminate the octant of $\theta_{23}$. The explanation of the anomaly in recent MINOS data (the difference of $\Delta m_{32}^{2}$ for neutrinos and antineutrinos), using long-range force originated from the mixing of the gauge boson $Z^{\prime}$ of $L_{\mu}-L_{\tau}$ with the standard model gauge boson $Z$, can be tested at ICAL at more than $5 \sigma \mathrm{CL}$. We have also discussed how to disentangle this from the solution with CPT violation using the seasonal change of the distance between the Earth and the Sun.
\end{abstract}

PACS numbers: 14.60.Pq

\section{INTRODUCTION}

The large hadron collider at CERN will probe the extensions of standard model above the electroweak scale. On the other hand, some of the extensions below the electroweak scale can be probed at the neutrino oscillation experiments. The extensions below the electroweak scale introduce massless or nearly massless gauge [1, 2] or Higgs bosons [3-6] and they couple with matter very feebly and remain invisible. These lead to the existence of new kind of forces either i) generating deviations of gravitational law at short distances, or ii) predicting low mass particles

*E-mail address: abhijit.samanta@gmail.com ${ }^{\dagger}$ past address where the work was initiated. whose exchange will induce forces at long distances, generally violating the equivalence principle $[7,8]$. A number of experiments have been searching for these new forces. The null results provide bounds on particle physics models, gravitational physics, and even on cosmological models [8-10].

The bounds on these couplings to baryon and/or lepton number [11] can be obtained from the testing of equivalence principle [12] (the free fall acceleration is same for all bodies independent of their chemical content). In [13], the author has used this idea to establish a bound on the strength of an hypothetical vectorial leptonic force and obtained the bound on the "fine structure" constant $\alpha \lesssim 10^{-49}$. See [14] for a review. A comparable limit also comes from lunar laser ranging [10], which measures the differential acceleration of the Earth and the Moon towards the 
Sun.

However, one can extend the SM with an additional $U(1)$ gauge symmetry without introducing any anomaly for one of the lepton flavor combinations: $L_{e}-L_{\mu}, L_{\mu}-L_{\tau}$, and $L_{e}-L_{\tau}$. The masses of the gauge bosons can be so light that the induced forces may have terrestrial range. Then the electrons inside the Sun can induce forces on the Earth surface depending on the lightness of the gauge boson. These forces on the Earth may also be from supernova neutrinos, galactic electrons depending on its ranges. These couple only to electron (and neutrino) density inside a massive object. As a result, the acceleration experienced by an object depends on its leptonic content and mass; and thus violates equivalence principle.

The long-range (LR) forces can play role in neutrino oscillation. The relatively stronger bounds than those from testing of the equivalence principle have been obtained from solar, atmospheric and supernova data $[15-19]^{1}$. The long-range forces due to galactic electrons, which can affect the galactic rotation curves if the range $R_{L R} \gtrsim R_{\text {gal }}$ (where $R_{\text {gal }}$ is the distance from the galactic center $\sim 10 \mathrm{kpc}$ ), is also now very tightly constrained [16]. Finally, all these results are consistent with the existing bounds on violation of equivalence principle.

The sign of this potential is opposite for neutrinos and anti-neutrinos and hence can lead to apparent differences in neutrino and anti-neutrino oscillation probabilities without introducing $\mathrm{CP}$ or $\mathrm{CPT}$ violation. For instance, the recently found discrepancy in the survival probabilities of $\nu_{\mu} \mathrm{s}$ and $\bar{\nu}_{\mu} \mathrm{s}$ in the MINOS experiment [21] has been explained using the mixing of $Z^{\prime}$ boson of $L_{\mu}-L_{\tau}$ symmetry with the $Z$ boson of the SM model; and the required associated parameters to explain this anomaly can be found in

\footnotetext{
1 There are plenty relic neutrinos and antineutrinos in the universe $\left(\sim 50 / \mathrm{cm}^{3}\right)$, which may screen the leptonic charges of the of celestial bodies. However, it has been analyzed in detail and shown that the screening is impossible [20]
}

$[22]$.

We have studied the long-range forces with atmospheric neutrinos at the magnetized iron calorimeter detector (ICAL) proposed at the India-based neutrino Observatory (INO) [23], which can directly measure the potential detecting separately $\nu_{\mu}$ s and $\bar{\nu}_{\mu} \mathrm{s}$. In this article we have focussed on:

- the bounds on the couplings of long-range forces,

- how significantly long-range forces modify the sensitivity of the measurement of oscillation parameters; particularly, the octant of 2-3 mixing (sign of $\delta^{\text {oct }}=\theta_{23}-45^{\circ}$ ) and the mass hierarchy (sign of $\left.\Delta m_{31}^{2}\right)$,

- test of the explanation of the MINOS data with long-range forces [22].

- possibility to disentangle the effect of CPT and long-range forces ${ }^{2}$.

We have also studied the changes of sensitivity to mass hierarchy and to $\theta_{23}$-octant with true (input) $\theta_{23}$ values considering no such forces (This was not studied in earlier works.).

The paper is organized as follows. We discuss the full three flavor neutrino oscillation in matter with long-range potential in sec. II, the details of the analysis method in sec. III, and the bounds on the couplings in Sec. IV. The effects of the long-range potential on discrimination of mass hierarchy and on $\theta_{23}$-octant is discussed in sec. V. Finally, the testing of the explanation of the anomaly in MINOS data using long-range force is described in Sec. VI. The discussion and conclusion are given in VII.

\footnotetext{
2 The advantages of atmospheric neutrinos to discriminate $C P T$ violation from $C P$ violation and nonstandard interactions have been discussed in [24].
} 


\section{OSCILLATION IN PRESENCE OF LONG-RANGE POTENTIAL}

The electrons inside the Sun generate a potential at the Earth by [15]:

$$
V_{e \mu, e \tau}=\alpha_{e \mu, e \tau} \frac{N_{e}}{R_{E S}} \approx 1.3 \times 10^{-11} \mathrm{eV}\left(\alpha_{e \mu, e \tau} / 10^{-50}\right),
$$

where, $\alpha_{e \mu, e \tau}=g_{e \mu, e \tau}^{2} / 4 \pi ; g_{e \mu, e \tau}$ are the gauge couplings of $L_{e}-L_{\mu}$ and $L_{e}-L_{\tau}$ symmetries. $N_{e} \approx 10^{57}$ is the number of electrons inside the Sun [25] and $R_{E S}=7.6 \times 10^{26} \mathrm{GeV}^{-1}$, the distance between the Earth and the Sun.

In a three neutrino framework the neutrino flavor states $\left|\nu_{\alpha}\right\rangle, \alpha=e, \mu, \tau$ can be expressed as linear superpositions of the neutrino mass eigenstates $\left|\nu_{i}\right\rangle$, $i=1,2,3$ with masses $m_{i}$ :

$$
\left|\nu_{\alpha}\right\rangle=\sum_{i} U_{\alpha i}\left|\nu_{i}\right\rangle .
$$

$U$ is the $3 \times 3$ unitary matrix. The time evolution of the flavor states is

$$
i \frac{\mathrm{d}}{\mathrm{dt}}\left[\nu_{\alpha}\right]=\frac{1}{2 E} U M_{\nu}^{2} U^{\dagger}\left[\nu_{\alpha}\right],
$$

where, $\left[\nu_{\alpha}\right]$ is the vector of flavor eigenstates, and $\left[\nu_{\alpha}\right]^{T}=\left[\left|\nu_{e}\right\rangle,\left|\nu_{\mu}\right\rangle,\left|\nu_{\tau}\right\rangle\right]$.

The evolution equation in the presence of matter and long-range potential is

$i \frac{\mathrm{d}}{\mathrm{d} t}\left[\nu_{\alpha}\right]=\frac{1}{2 E}\left[U M_{\nu}^{2} U^{\dagger}+\left(\begin{array}{ccc}A & 0 & 0 \\ 0 & 0 & 0 \\ 0 & 0 & 0\end{array}\right)+V_{L R}\right]\left[\nu_{\alpha}\right]$

where,

$$
V_{L R}=\left(\begin{array}{ccc}
V_{e \mu} & 0 & 0 \\
0 & -V_{e \mu} & 0 \\
0 & 0 & 0
\end{array}\right) \text { or }\left(\begin{array}{ccc}
V_{e \tau} & 0 & 0 \\
0 & 0 & 0 \\
0 & 0 & -V_{e \tau}
\end{array}\right) .
$$

The matter term $A=2 \sqrt{2} G_{F} n_{e} E=7.63 \times$ $10^{-5} \mathrm{eV}^{2} \rho(\mathrm{gm} / \mathrm{cc}) E(\mathrm{GeV}) \mathrm{eV}^{2}$. Here, $G_{F}, n_{e}$ and $\rho$ are the Fermi constant, the electron number density and the matter density of the medium, respectively. The evolution equation for antineutrinos has the reversed sign for $A, V_{L R}$ and the phase $\delta$. We have numerically solved full three flavor oscillation in presence of matter and long-range forces.

However, to understand the bounds it is easy to consider two flavor $\mu-\tau$ oscillation, (which has been studied in [15] to constrain the long-range forces using atmospheric neutrino data of Super-Kamiokande experiment [26]). To understand the effect of 1-3 mixing, one needs to consider the changes of the effective oscillation parameters due to $V_{L R}$. The $\mu-\tau$ oscillation in presence of $V_{e \tau}$ is governed by the evolution equation

$$
\begin{aligned}
& i \frac{d}{d t}\left(\begin{array}{c}
\nu_{\mu} \\
\nu_{\tau}
\end{array}\right)= \\
& \left(\begin{array}{cc}
-\frac{\Delta m_{32}^{2}}{4 E} \cos 2 \theta_{23} & \frac{\Delta m_{32}^{2}}{4 E} \sin 2 \theta_{23} \\
\frac{\Delta m_{32}^{2}}{4 E} \sin 2 \theta_{23} & \frac{\Delta m_{32}^{2}}{4 E} \cos 2 \theta_{23}-V_{e \tau}
\end{array}\right)\left(\begin{array}{l}
\nu_{\mu} \\
\nu_{\tau}
\end{array}\right)(6)
\end{aligned}
$$

Then, the survival probability of $\nu_{\mu}$

$$
P_{\mu \mu}=1-\sin ^{2} 2 \tilde{\theta}_{23} \sin ^{2} \frac{\Delta \tilde{m}_{23}^{2} L}{4 E},
$$

where, $L$ is the neutrino flight path length. The effective mixing angle $\tilde{\theta}_{23}$ and $\Delta \tilde{m}_{32}^{2}$ are related with their vacuum quantities by the relations

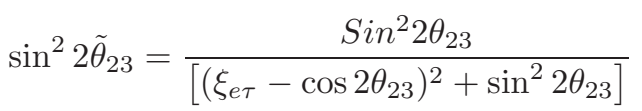

and

$$
\left.\Delta \tilde{m}_{23}^{2}=\Delta m_{23}^{2}\left[\left(\xi_{e \tau}-\cos 2 \theta_{23}\right)^{2}+\sin ^{2} 2 \theta_{23}\right)^{1 / 2}\right] ;
$$

where, $\xi_{e \tau} \equiv \frac{2 V_{e \tau} E}{\Delta m_{32}^{2}}$. The potential $V_{e \tau}$ and the corresponding $\xi$ change sign for $\bar{\nu}$. Similarly, for $L_{e}-L_{\mu}$ gauge symmetry the survival probability can be obtained and they satisfy

$$
P_{\mu \mu}\left(V_{e \tau}\right)=P_{\bar{\mu} \bar{\mu}}\left(-V_{e \tau}\right)=P_{\mu \mu}\left(-V_{e \mu}\right)=P_{\bar{\mu} \bar{\mu}}\left(V_{e \mu}\right)
$$

\section{THE $\chi^{2}$ ANALYSIS}

To evaluate the potential of ICAL with atmospheric neutrinos we generate events by NUANCE-v3 [27] and consider only the muon energy and its direction (directly measurable quantities) of the events for 
a conservative estimation. The energy and angular resolutions of the muons at ICAL are very high: 4$10 \%$ for energy and $4-12 \%$ for zenith angle, which are obtained from GEANT [28] simulation. The ranges are due to different energies and different angles with respect to the iron layers. These uncertainties are very negligible compared to the uncertainties in reconstructed neutrinos due to kinematics of the scattering processes.

The major uncertainty arises from the particles produced in the event other than muon. One might expect that consideration of the hadrons for neutrino energy $\gtrsim 2 \mathrm{GeV}$ will improve the sensitivities substantially. But, in [29], it has been found that there is a very marginal improvement on measurement of $\theta_{23}$ and a small improvement on $\Delta m_{31}^{2}$ : $\delta\left(\Delta m_{31}^{2}\right)=0.02 \times 10^{-3} \mathrm{eV}^{2}$. The fact is that the total hadron energy is carried out by multiple low energy hadrons. The average energy per hadron is $\lesssim 1 \mathrm{GeV}$ and the average number of hadrons per event is $\gtrsim 2$. The energy resolution of the hadrons at this energy is $\sim 80 \%$, and consequently, the neutrino energy resolutions do not improve significantly after adding the hadrons ${ }^{3}$.

The $\chi^{2}$ is calculated according to the Poisson probability distribution with flat uncertainties of the oscillation parameters. The term due to the contribution of prior information of the oscillation parameters measured by other experiments is not added to $\chi^{2}$ to examine solely the performance of ICAL. The data have been binned in cells of equal size in the $\log _{10} E$ - $L^{0.4}$ plane, where $L=2 R \cos \theta_{Z}$. The choice of binning is motivated by pattern of the oscillation probability $P\left(\nu_{\mu} \rightarrow \nu_{\mu}\right)$ in the $L-E$ plane [30]. The distance between two consecutive oscillation peaks driven by $\Delta m_{23}^{2}$ increases (decreases) as one goes to lower $L(E)$ values for a given $E(L)$. The binning

\footnotetext{
3 The detection of the neutral current events may also be possible, which have no directional information, but the energy dependence of the oscillation averaged over all directions can contribute to the total $\chi^{2}$ in the sensitivity studies.
}

of $L$ has been optimized to get better sensitivity to the oscillation parameters. To maintain $\chi^{2} /$ d.o.f $\approx 1$ for Monte Carlo simulation study, number of events should be $>4$ per cell [31] (as large number of cells at high energies have number of events less than 4 or even zero and they increase the $\chi^{2} /$ d.o.f substantially beyond 1 ). If the number is less than 4 (which happens in the high energy bins), we combine events from the nearest cells.

The migration of the number of events from true neutrino energy and zenith angle cells to muon energy and zenith angle cells is made using exact energyangle correlated 2-dimensional resolution functions [32]. For each set of oscillation parameters, we integrate the oscillated atmospheric neutrino flux folding with the cross section, the exposure time, the target mass, the efficiency and the two dimensional energyangle correlated exact resolution functions to obtain the predicted data in each cell in $L-E$ plane for the $\chi^{2}$ analysis. We use the charge current cross section of Nuance-v3 [27] and the Honda flux in 3-dimensional scheme [33]. The number of bins and resolution functions have been optimized in [31]. Both theoretical (fit values) and experimental (true values) data for $\chi^{2}$ analysis have been generated in the same way by migrating number of events from neutrino to muon energy and zenith angle bins using the resolution functions [34].

The systematic uncertainties of the atmospheric neutrino flux are crucial for determination of the oscillation parameters. We have divided them into two categories: (i) the overall flux normalization uncertainties which are independent of the energy and zenith angle, and (ii) the spectral tilt uncertainties which depend on $E$ and $\theta_{\mathrm{z}}$.

The flux with uncertainties included can be written as

$$
\begin{aligned}
& \Phi\left(E, \theta_{Z}\right)=\Phi_{0}(E)\left[1+\delta_{E} \log _{10} \frac{E}{E_{0}}\right] \\
& \times\left[1+\delta_{Z}\left(\left|\cos \theta_{Z}\right|-0.5\right)\right] \times\left[1+\delta_{f_{N}}\right]
\end{aligned}
$$

For $E<1 \mathrm{GeV}$ we take the energy dependent uncertainty $\delta_{E}=15 \%$ and $E_{0}=1 \mathrm{GeV}$ and for $E>10$ 
$\mathrm{GeV}, \delta_{E}=5 \%$ and $E_{0}=10 \mathrm{GeV}$. The overall flux uncertainty as a function of zenith angle is parametrized by $\delta_{Z}$. According to [33] we use $\delta_{Z}=4 \%$, which leads to $2 \%$ vertical/horizontal flux uncertainty. We take the overall flux normalization uncertainty $\delta_{f_{N}}=$ $10 \%$ and the overall neutrino cross-section uncertainty $\delta_{\sigma}=10 \%$.

We evaluate the $\chi^{2}$ for both normal hierarchy $(\mathrm{NH})$ and inverted hierarchy (IH) with $\nu$ s and $\bar{\nu}$ s separately for a given set of oscillation parameters. Then we find the total $\chi^{2}\left(=\chi_{\nu}^{2}+\chi_{\bar{\nu}}^{2}\right)$.

We have set the inputs of $\left|\Delta m_{32}^{2}\right|=2.5 \times$ $10^{-3} \mathrm{eV}^{2}$, and $\delta_{C P}=0$. We marginalize $\chi^{2}$ over $\Delta m_{32}^{2}, \theta_{23}, \theta_{13}$ and $\alpha$. We have chosen the range of $\Delta m_{32}^{2}=2.0-3.0 \times 10^{-3} \mathrm{eV}^{2}, \theta_{23}=37^{\circ}-54^{\circ}$, $\theta_{13}=0^{\circ}-12.5^{\circ}$, and $\alpha_{e \mu, e \tau}=0-3 \times 10^{-53}$. The solar parameters are fixed at their best-fit values: $\Delta m_{21}^{2}=7.67 \times 10^{-5} \mathrm{eV}^{2}$ and $\sin ^{2} \theta_{12}=0.312$ [35]. The effect of $\Delta m_{21}^{2}$ comes in sub-leading order in the oscillation probability for atmospheric neutrinos when $E \gtrsim \mathrm{GeV}$ and it is very negligible.

In this analysis we have considered an exposure of 1 Mton.year (which is 10 years run of $100 \mathrm{kTon}$ ) of ICAL and energy range $0.8-15 \mathrm{GeV}$.

\section{BOUNDS ON COUPLINGS}

For atmospheric neutrinos at the magnetized ICAL the bounds on $\alpha_{e \mu}$ and $\alpha_{e \tau}$ are shown in the left pannel in Fig. 1 and its strength to constrain them with upper as well as lower bounds are shown in the right pannel in Fig. 1 with a typical nonzero input (true) value. We have checked that the bounds do not change significantly for different combinations of true values of oscillation parameters.

The bounds $\alpha_{e \mu} \lesssim 5.5 \times 10^{-52}$ and $\alpha_{e \tau} \lesssim 6.4 \times$ $10^{-52}$ at $90 \%$ CL have been obtained from present atmospheric neutrino oscillation data [15]. Considering both atmospheric and solar data the bounds are $\alpha_{e \mu} \lesssim 3.4 \times 10^{-53}$ and $\alpha_{e \tau} \lesssim 2.5 \times 10^{-53}$ at $3 \sigma \mathrm{CL}$ [16]. The better precision and smaller value by a few order of magnitude of solar mass squared difference than the atmospheric one play here the main role to make it significantly stringent.

The bounds are relatively stronger at the magnetized detector: $\alpha_{e \mu, e \tau} \lesssim 1.65 \times 10^{-53}$ at $3 \sigma \mathrm{CL}$. For atmospheric neutrinos it comes mainly due to the high precision of $\Delta m_{32}^{2}$ and this can be understood quite well from simple two flavor survival probability of $\nu_{\mu}$ and $\bar{\nu}_{\mu}$ in vacuum with effective $\Delta m_{32}^{2}$ and $\theta_{23}$ for long-range potentials. This becomes possible due to the fact that the 1-3 mixing effect is sub-leading (as $\left.\theta_{13}<11.38^{\circ}[36]\right)$ and it can be neglected at this moment for simplicity. When the potential $V_{L R}$ comes to the play, it tries to change the effective value of $\Delta m_{31}^{2}$ (see Eq. 9) and becomes tightly constrained. The sign $V_{L R}$ is opposite for $\nu$ and $\bar{\nu}$, and ICAL can detect them separately. This makes the bounds more tighter at ICAL and lessens the difference in bounds between $\alpha_{e \mu}$ and $\alpha_{e \tau}$, while the difference is substantially large at non-magnetized detectors.

\section{EFFECTS ON 2-3 SECTOR}

\section{A. Determination of mass hierarchy}

In Fig. 2 we show the sensitivity to mass hierarchy for different true values of $\theta_{23}$. We show it for three cases: assuming no potential for long-range forces $\left(\alpha_{L R}=0\right)$ and with a benchmark input (true) value for $\alpha_{e \mu}$ and $\alpha_{e \tau}$, respectively.

For all three cases the sensitivity increases as one goes to the higher $\theta_{23}$ values and it is a general feature for both cases with and without the potential for longrange force. This can be understood from the $\nu_{\mu}$ flux at the detector considering the effect of 1-3 mixing. Here we assume no potential for long-range force. The ratio of the $\nu_{\mu}$ flux at the detector (oscillated) and at the source (original) [29]:

$$
\frac{F_{\mu}}{F_{\mu}^{0}} \approx K\left(\sin 2 \theta_{23}\right)-f\left(\theta_{23}\right)\left(1-\frac{1}{r}\right) P_{A}\left(\theta_{13}\right),
$$

where, $K\left(\sin 2 \theta_{23}\right)$ is an even function of the deviation 

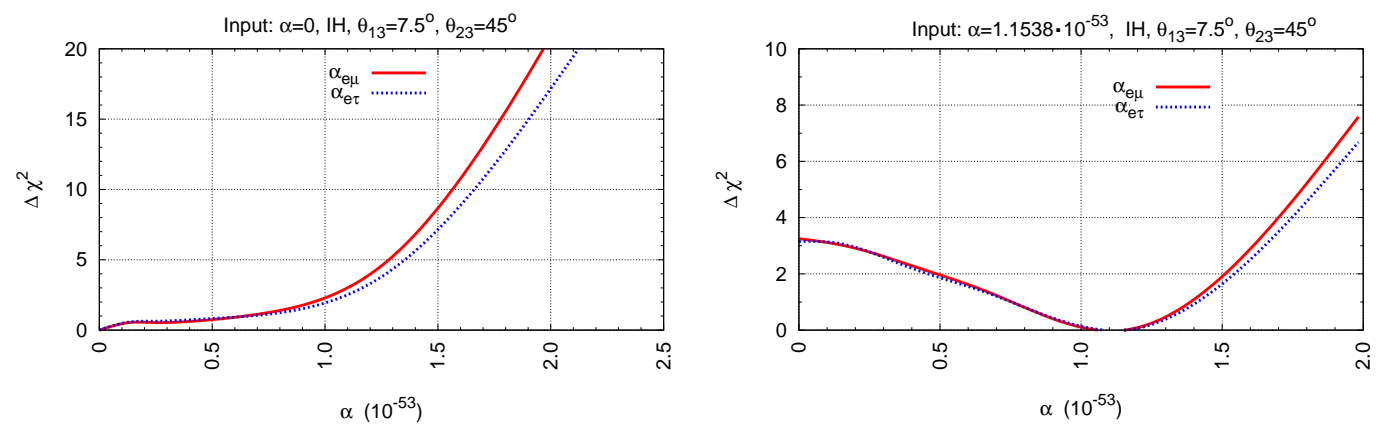

FIG. 1: The upper bounds of $\alpha_{e \mu}$ and $\alpha_{e \tau}$ for true (input) $\alpha=0$ in the left pannel; and the corresponding upper as well as lower bounds for input $\alpha=1.1538 \times 10^{-53}$ in the right pannel, respectively.
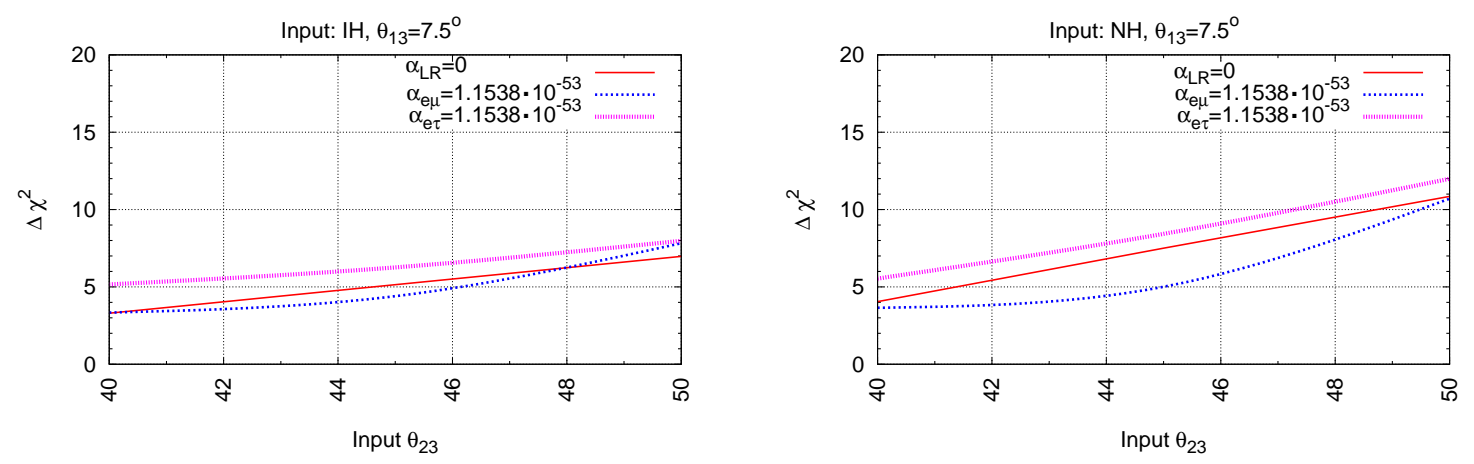

FIG. 2: The sensitivities to mass hierarchy for different true (input) $\theta_{23}$ values for input IH (left) and input NH (right), respectively. For each case we have considered true (input) values of $\alpha_{L R}=0, \alpha_{e \mu}=1.1538 \times 10^{-53}$, and $\alpha_{e \tau}=$ $1.1538 \times 10^{-53}$, respectively.

(symmetric with respect to change of the octant), and $P_{A}\left(\theta_{13}\right)$ is a function of $\theta_{13}$ only, and

$$
f\left(\theta_{23}\right) \equiv\left(s_{23}^{4}-\frac{s_{23}^{2}}{r}\right)
$$

which increases quickly with $\theta_{23}$, so that for $r=3-4$, $f\left(\theta_{23}=40^{\circ}\right) \ll f\left(\theta_{23}>50^{\circ}\right)$. Therefore, for $\theta_{23}<45^{\circ}$ the flux $F_{\mu}$ has much weaker dependence on $\theta_{13}$ than for $\theta_{23}>45^{\circ}$. This is reflected in the sensitivity to mass hierarchy as the lower limit of $\theta_{13}$ has been taken zero during marginalization and no prior contribution from other future experiments has been considered. However, in future the lower limit will be known from other experiments (as very recently T2K puts a lower bound [37]); then the $\theta_{23}$-dependence will be less and the sensitivity to mass hierarchy will also be substantially improved [34].

The potential $V_{e \mu}$ opposes, while $V_{e \tau}$ helps to determine the hierarchy (see Fig. 2). As the 1-3 mixing is small $\left(\theta_{13}<11.2^{\circ}\right)$, the potential $V_{e \tau}$ decreases the effective value of $\Delta m_{31}^{2}$. Consequently, the resonant energy decreases (see Fig. 2 of [32]), where the atmospheric neutrino flux is relatively large and it helps in determination of hierarchy. On the other hand, as 2-3 mixing is large, $V_{e \mu}$ increases the effective value of $\Delta m_{31}^{2}$ for $\theta_{23}>45^{\circ}$ (see Eq. 9). In spite of an enhancement in 1-3 mixing (which happens for both potentials) the final sensitivity decreases for $V_{e \mu}$ due to relatively low statistics at the resonant zones. These happen for $\nu$ with $\mathrm{NH}$ and for $\bar{\nu}$ with $\mathrm{IH}$. 

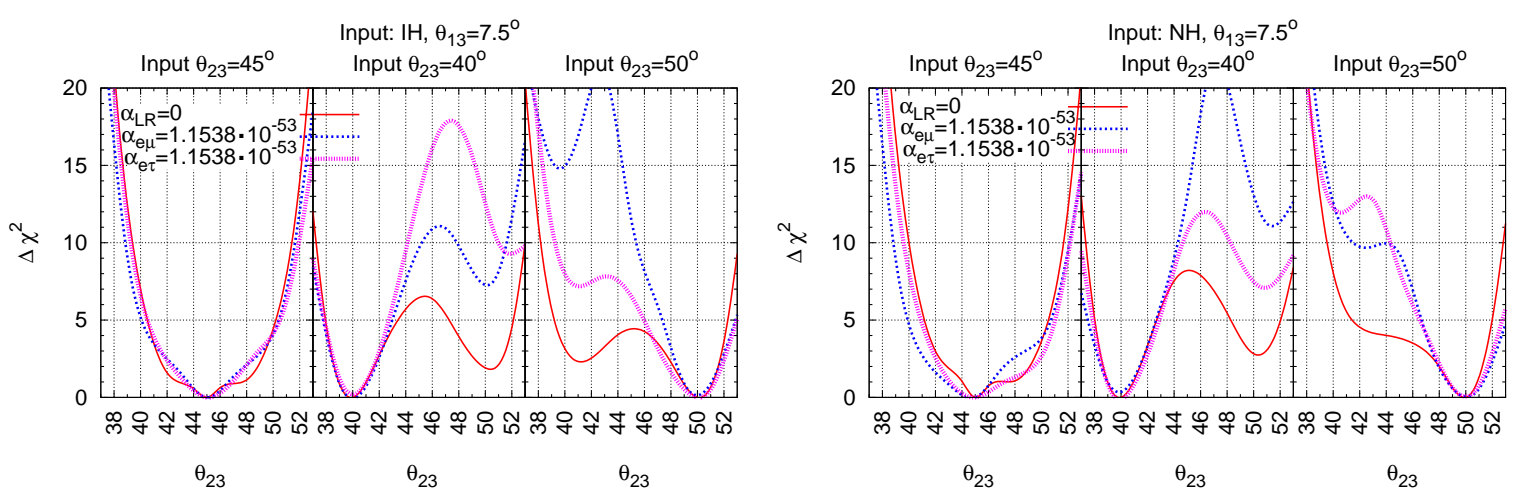

FIG. 3: The sensitivities to deviation of 2-3 mixing from maximal mixing as well as its octant for both true (input) IH (left) and $\mathrm{NH}$ (right). For each case we have considered $\alpha_{L R}=0, \alpha_{e \mu}=1.1538 \times 10^{-53}, \alpha_{e \tau}=1.1538 \times 10^{-53}$, respectively.

\section{B. Determination of octant of $\theta_{23}$}

The sensitivity to 2-3 mixing, mainly the deviation from its maximal mixing $\left(\delta=\theta_{23}-45^{\circ}\right)$ and the octant ( $\operatorname{sign}$ of $\delta$ ) are shown in Fig. 3 for three cases: i) $\alpha_{L R}=0$, ii) $\alpha_{e \mu}=1.1538 \times 10^{-53}$, and iii) $\alpha_{e \tau}=1.1538 \times 10^{-53}$, respectively. It is important to note here that octant discrimination is significantly improved in presence of long-range potentials. This is due to the fact that effective 1-3 mixing is always larger for both potentials. For a given hierarchy the change of the sensitivity with the change of true (input) octant depends mainly on the magnitude of $\delta^{\mathrm{eff}}=\theta_{23}{ }^{\mathrm{eff}}-45^{\circ}$, which again strongly depends on the potentials (see Eq. 8). The change of the effective value of $\Delta m_{31}^{2}$ works here subdominantly (while it was a dominating factor for determination of the mass hierarchy). Now, for $V_{e \tau}$ with $\mathrm{NH}$, sensitivity to octant determination is better for $\theta_{23}>45^{\circ}$ than $\theta_{23}<45^{\circ}$ as $\delta^{\text {eff }}$ is increased due to $V_{e \tau}$ for neutrinos and the flux is two times higher for neutrinos than antineutrinos. This is opposite for $V_{e \mu}$ : octant determination is better for $\theta_{23}<45^{\circ}$ than $\theta_{23}>45^{\circ}$. Similarly, the results with IH can be understood using antineutrinos considering the symmetries in Eq. 10.

\section{MINOS ANOMALY}

The anomaly in recent MINOS data (difference in measured values of 2-3 mass splittings and mixing angles for $\nu$ and $\bar{\nu}[21]$ ), which implies CPT violation or signal for non-standard interactions, has been explained by long-range potential due to $L_{\mu}-L_{\tau}$ gauge symmetry [22]. The Sun does not contain any $\mu$ or $\tau$ and hence there is no direct bound from neutrino oscillation. However, the potential can be induced indirectly on the Earth by the mixing of the gauge boson $Z^{\prime}$ of $L_{\mu}-L_{\tau}$ with the standard model $Z$ boson [22]:

$$
V_{\mu \tau}=3.6 \times 10^{-14} \mathrm{eV}\left(\frac{\alpha_{\mu \tau}}{10^{-50}}\right)
$$

The sign changes for $\bar{\nu}$. From the analysis of atmospheric neutrino data at ICAL with an exposure of 1 Mton.yr, we find the bound on the coupling $\alpha_{\mu \tau} \geq 3.2(4.2) \times 10^{-51}$ at $3(5) \sigma \mathrm{CL}$ and the explanation of MINOS data [22] can be tested at more than $5 \sigma \mathrm{CL}$.

\section{DISCUSSION AND CONCLUSION}

In this paper, we have estimated the conservative bounds on long-range forces with atmospheric neutrinos at ICAL: $\alpha_{e \mu, e \tau} \lesssim 1.65 \times 10^{-53}$ at $3 \sigma$ CL. This 
bounds are significantly stronger than the present bounds.

The CPT violation and the long-range forces, which are the candidates for solution of recent anomaly in MINOS data, can be discriminated with atmospheric neutrinos at ICAL. The distance between the Sun and the Earth varies and the difference between aphelion and perihelion is about $3 \%$, which causes seasonal variation of the long-range forces. But, there should not be any such change for CPT violation.

Assuming one by one nonzero input value of these couplings it is found that while the potential $V_{e \mu}$ opposes; $V_{e \tau}$ helps to discriminate the mass hierarchy. However, both potentials help to discriminate octant of $\theta_{23}$. The anomaly of MINOS data, which has been explained using long-range force potential, can be tested at ICAL with more that $5 \sigma$ CL.

Acknowledgements: This work was started at Harish-Chandra Research Institute and has been supported partly by the Neutrino Physics projects of this institute. The use of cluster computational facility installed by the funds of this project is gratefully acknowledged.
[1] R. Foot, Mod. Phys. Lett. A 6, 527 (1991); X.-G. He, G. C. Joshi, H. Lew and R. R. Volkas, Phys. Rev D 43, 22 (1991).

[2] M. Dine, W. Fischler and M. Srednicki, Phys. Lett. B 104, 199 (1981); J. E. Kim, Phys. Rev. Lett., 43 103 (1979).

[3] Y. Chikashige, R. N. Mohapatra and R. D. Peccei, Phys. Lett. B 98, 265 (1981).

[4] G. B. Gelmini and M. Roncadelli, Phys. Lett. B 99, 411 (1981).

[5] L. J. Hall and S. J. Oliver, Nucl. Phys. Proc. Suppl. 137, 269 (2004) [arXiv:hep-ph/0409276] and references therein.

[6] D. Chang, W. Y. Keung and P. B. Pal, Phys. Rev. D 42, 630 (1990).

[7] T. Damour, Class. Quant. Grav. 13, A33 (1996) [arXiv:gr-qc/9606080].

[8] Fischbach E, Gillies G T, Krause D E, Schwan J G and Talmadge C 1992 Metrologia 29213

[9] E. G. Adelberger, B. R. Heckel and A. E. Nelson, Ann. Rev. Nucl. Part. Sci. 53, 77 (2003) [arXiv:hep$\mathrm{ph} / 0307284]$.

[10] J. G. Williams, X. X. Newhall and J. O. Dickey, Phys. Rev. D 53, 6730 (1996).

[11] T. D. Lee and C. N. Yang, Phys. Rev. 98 (1955) 1501.

[12] R. V. Eotvos, D. Pekar, E. Fekete, Annalen Phys. 68, 11-66 (1922).

[13] L. Okun, Phys. Lett. B 382, 389 (1996) [arXiv:hep$\mathrm{ph} / 9512436]$.

[14] A. D. Dolgov, Phys. Rept. 320, 1 (1999).
[15] A. S. Joshipura and S. Mohanty, Phys. Lett. B 584, 103 (2004) [arXiv:hep-ph/0310210].

[16] A. Bandyopadhyay, A. Dighe and A. S. Joshipura, Phys. Rev. D 75, 093005 (2007) [arXiv:hepph/0610263].

[17] M. C. Gonzalez-Garcia, P. C. de Holanda, E. Masso and R. Zukanovich Funchal, JCAP 0701, 005 (2007) [arXiv:hep-ph/0609094].

[18] J. A. Grifols, E. Masso and S. Peris, Astropart. Phys. 2, 161 (1994).

[19] J. A. Grifols and E. Masso, Phys. Lett. B 579, 123 (2004) [arXiv:hep-ph/0311141].

[20] S. I. Blinnikov, A. D. Dolgov, L. B. Okun and M. B. Voloshin, Nucl. Phys. B 458, 52 (1996) [arXiv:hep-ph/9505444].

[21] Talk given by P. Vahle at 24th International Conference On Neutrino Physics And Astrophysics (Neutrino 2010), June 14, 2010.

[22] J. Heeck and W. Rodejohann, arXiv:1007.2655 [hepph]; H. Davoudiasl, H. -S. Lee, W. J. Marciano, [arXiv:1102.5352 [hep-ph]].

[23] V. Arumugam et al. [INO Collaboration], [ See, http://www.imsc.res.in/ ino ]

[24] A. Samanta, Phys. Lett. B693, 296-300 (2010). [arXiv:1005.4851 [hep-ph]].

[25] John N. Bahcall, Neutrino Astrophysics (Cambridge Univ. Press, Cambridge, England, 1989).

[26] Y. Fukuda et al. Phys Rev Lett. 82, 2644 (1999); Y. Fukuda et al. Phys Lett . B388, 397 (1999);Y. Fukuda et al. Phys Rev Lett. 85, 3999 (2000). 
[27] D. Casper, Nucl. Phys. Proc. Suppl. 112, 161 (2002) [arXiv:hep-ph/0208030].

[28] http://geant4.web.cern.ch/geant4/

[29] A. Samanta, A. Y. .Smirnov, JHEP 1107, 048 (2011). [arXiv:1012.0360 [hep-ph]].

[30] A. Samanta, Phys. Rev. D 79, 053011 (2009) [arXiv:0812.4640 [hep-ph]].

[31] A. Samanta, Phys. Rev. D 80, 113003 (2009) [arXiv:0812.4639 [hep-ph]].

[32] A. Samanta, Phys. Lett. B 673, 37 (2009) [arXiv:hep$\mathrm{ph} / 0610196]$.

[33] M. Honda, T. Kajita, K. Kasahara, S. Midorikawa and T. Sanuki, Phys. Rev. D 75, 043006 (2007) [arXiv:astro-ph/0611418].

[34] A. Samanta, Phys. Rev. D81, 037302 (2010). [arXiv:0907.3540 [hep-ph]].

[35] G. L. Fogli et al., Phys. Rev. D 78, 033010 (2008) [arXiv:0805.2517 [hep-ph]].

[36] T. Schwetz, M. Tortola, J. W. F. Valle, New J. Phys. 13, 063004 (2011). [arXiv:1103.0734 [hep-ph]].

[37] K. Abe et al. [ T2K Collaboration ], [arXiv:1106.2822 [hep-ex]]. 